\title{
Millimetric Sardinia radio Telescope Receiver based on Array of Lumped elements kids
}

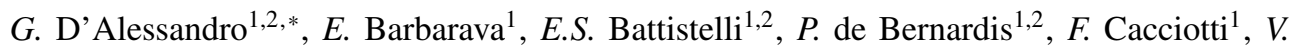
Capalbo $^{1}, E$. Carretti ${ }^{3}, F$. Columbro ${ }^{1,2}, A$. Coppolecchia ${ }^{1,2}, A$. Cruciani $^{2}, M$. De Petris ${ }^{1,2}, F$. Govoni $^{4}, G$. Isopi ${ }^{1}, L$. Lamagna ${ }^{1,2}, P$. Marongiu $^{4}, S$. Masi $^{1,2}, L$. Mele $^{1,2}, M$. Molinari $^{4}, M$. Murgia $^{4}, A$. Navarrini ${ }^{4}, A$. Orlati ${ }^{3}, A$. Paiella ${ }^{1,2}, G$. Pettinari ${ }^{5}, F$. Piacentini ${ }^{1,2}, T$. Pisanu ${ }^{4}, S$. Poppi $^{4}, G$. Presta ${ }^{1,2}$, and $F$. Radiconi ${ }^{1,2}$

${ }^{1}$ Sapienza Università di Roma, Roma, Italy

${ }^{2}$ INFN sezione di Roma, 00185 Roma, Italy

${ }^{3}$ INAF - Istituto di Radioastronomia

${ }^{4}$ INAF - Osservatorio Astronomico di Cagliari

${ }^{5}$ CNR-IFN - Istituto di Fotonica e Nanotecnologie, Roma, Italy

\begin{abstract}
MISTRAL is a millimetric camera working in the W-band (78-103 GHz) which will take data from the Sardinia Radio Telescope, the Italian 64-m radio telescope located $50 \mathrm{~km}$ form Cagliari, at $600 \mathrm{~m}$ above the sea level, in Sardinia. It is being built as a facility instrument by the Sapienza University for INAF, that manages the radio telescope, under a PON contract. It will consist of a compact cryostat hosting the re-imaging optics, cooled at $4 \mathrm{~K}$, and a 408-pixel array of photon-noise limited lumped element kinetic inductance detectors fabricated at CNR-IFN and cooled at a base temperature lower than $300 \mathrm{mK}$. MISTRAL will be able to investigate a long list of scientific targets spanning from extragalactic astrophysics to solar system science, with high angular resolution $(\sim 12$ arcsec), including Sunyaev Zel'dovich effect measurements and the study of the Cosmic Web.
\end{abstract}

\section{Introduction}

Sardinia Radio telescope (SRT) is a multipurpose telescope aimed to measure the electromagnetic radiation from $300 \mathrm{MHz}$ to $116 \mathrm{GHz}$. After the scientific commissioning, in 2015, 4 receivers have been installed and successfully operated at frequency lower than $26.5 \mathrm{GHz}$. New instruments are going to be installed in the next future, among which, MISTRAL will measure in the $\mathrm{W}$-band.

High angular resolution W-band measurements open at a number of possibilities of scientific cases, including galaxies spectral energy distribution measurement and their continuum observation [1], AGN and radio galaxies [2], detection of circumstellar disks [3], dense core in giant molecular gas investigation [4], synchrotron non thermal jet and hot spot [5], intraclusters filaments, cosmic web, and other S-Z related observation [6, 7]; and even more, by correlating with other experiment.

\footnotetext{
*e-mail: giuseppe.dalessandro@ roma1.infn.it
} 
Because of the large diameter telescope needed to perform high angular resolution measurements only few cameras around the world are devoted to this, such as MUSTANG2 [8] at the Green Bank Telescope.

MISTRAL aims to perform high angular resolution measurement with an array of lumped element kids detectors coupled, thanks to a re-imaging optic system, with SRT. MISTRAL is in an advanced phase of manufacturing and all the parts of the experiment are going to be assembled together at the beginning of 2022. MISTRAL will be installed in March 2022 and commissioning activities will start in spring 2022.

In section 2 we describe the Sardinia Radio Telescope enlightening its optical system and the typical observational conditions. Section 3 describes the instrument and its main parts.

\section{Sardinia Radio Telescope}

SRT [9] is a multipurpose instrument operated in either single dish or Very Long Baseline Interferometer mode. It is located 50km north of Cagliari in Sardinia (Italy), Lat. 39.4930N - Long. 9.2451E, $600 \mathrm{~m}$ above sea level. Construction was completed in August 2012. The technical commissioning phase to validate scientific performances was managed by National Institute for Astrophysics and concluded in 2014. The Early Science Program observations started in 2016, and regular observations in 2018.

The primary antenna (M1) is fully steerable, $64 \mathrm{~m}$ in diameter. It is composed of 1008 aluminum elements controlled by electromechanical actuators. An f/0.33 primary focus occurs near the sub-reflector (M2), $7.9 \mathrm{~m}$ in diameter, composed of 49 aluminum elements. Its position can be changed for focus adjustment. M1 and M2 are shaped to minimize spillover and the standing waves between the feed and the sub-reflector. The Gregorian focus, $\mathrm{f} / 2.34$ occurs around 20 meters below M2 in the Gregorian room, the violet box in Figure 1. MISTRAL will be placed in this room by using the Gregorian focus of SRT.

SRT has been designed to perform measurements from $305 \mathrm{MHz}$ to $116 \mathrm{GHz}$ [10]. Four receivers have been successfully installed: L-band $(305 \mathrm{MHz}-410 \mathrm{MHz})$, P-band $(1.3 \mathrm{GHz}$ - 1.8 GHz), C-band $(5.7 \mathrm{GHz}-7.7 \mathrm{GHz})$ and $\mathrm{K}$-band $(18 \mathrm{GHz}-26.5 \mathrm{GHz})$. In the next future, new receivers will increase the frequency coverage [10]: Q-band $(33 \mathrm{GHz}-50 \mathrm{GHz})$, polarization-sensitive $\mathrm{W}$-band ( 16 beams), K/Q/W band (3 beams) polarization-sensitive, and MISTRAL ${ }^{1}$.

Table 1. SRT optical and mechanical main features

\begin{tabular}{ll}
\hline M1 diameter & $64 \mathrm{~m}$ \\
M2 diameter & $7.9 \mathrm{~m}$ \\
Primary focus & $\mathrm{f} / 0.33$ \\
Gregorian focus & $\mathrm{f} / 2.34$ \\
\hline Pointing accuracy & $2-13 \mathrm{arcsec}$ \\
Range in elevation & $5-90 \mathrm{deg}$ \\
Range in azimuth & $180 \pm 270 \mathrm{deg}$ \\
\hline
\end{tabular}

Among other activities, a metrological activity to measure with high precision deformations of the SRT due to gravitational and thermal effects is undergoing [10]; by means of the

\footnotetext{
${ }^{1}$ The Enhancement of the Sardinia Radio Telescope (SRT) for the study of the Universe at high radio frequencies is financially supported by the National Operative Program (Programma Operativo Nazionale - PON) of the Italian Ministry of University and Research Research and Innovation 2014-2020, Notice D.D. 424 of 28-02-2018 for the granting of funding aimed at strengthening research infrastructures, in implementation of the Action II.1 - Project Proposal PIR01_00010
} 


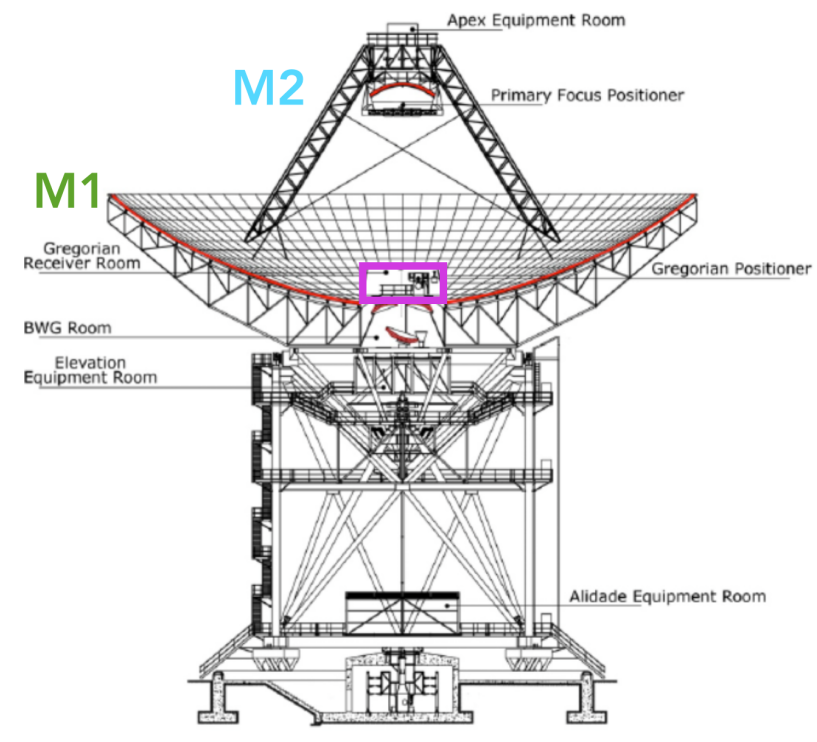

Figure 1. SRT schematic. The primary mirror (M1) consists of 1008 individual aluminum panels. Each panel has an area ranging from 2.4 to $5.3 \mathrm{~m}^{2}$. It is built using aluminum sheets glued with epoxy resin. The sub-reflector (M2) consists of 49 individual aluminum panels. Near M2 occurs a primary f/0.33 focus. MISTRAL will be placed in the Gregorian room (violet box) where the f/2.34 Gregorian focus occurs.

active surface it will be possible to correct the deformations of the main mirror allowing the surface roughness needed for observations up to $116 \mathrm{GHz}$. Also, the metrological system will allow high precision pointing measuring and correcting the subreflector displacement from the optical axis together with the deformation of the whole structure.

\subsection{Site condition}

Estimation of sky opacity, based on recorded atmospheric data, forecasts by Navarrini et al. (http://hdl.handle.net/20.500.12386/28787) report $\tau<0.15\left(50^{\text {th }}\right.$ percentile $)$ at $93 \mathrm{GHz}$ during the winter nights. The average PWV in the same conditions is mainly $8 \mathrm{~mm}$. 50 years of radiosonde profiles taken at Cagliari airport (30 km far, at sea level) and scaled for SRT site shows PWV $<11 \mathrm{~mm}\left(50^{\text {th }}\right.$ percentile $)$ and opacity $<0.2\left(50^{\text {th }}\right.$ percentile $)$ at $100 \mathrm{GHz}$ [11]. What mainly concern W-band measurement a dynamic scheduling will be applied to optimize the observational time and weather conditions.

\section{MISTRAL instrument}

MISTRAL is a W-band camera for SRT. The focal plane is composed of 408 Lumped element kinetic inductance detectors (LEKID), fabricated at CNR-IFN, arranged on a $97 \mathrm{~mm}$ circular focal plane. The focal plane is optically coupled with SRT thanks to a re-imaging optics system composed of two silicon lenses coated with an anti-reflection system. The focal plane is cooled down at $270 \mathrm{mK}$ with a cryostat provided by QMC instruments from Cardiff (UK). 


\subsection{Cryostat}

The cryostat is provided by QMC instruments ${ }^{2}$. It is composed of two radiation shields at $40 \mathrm{~K}$ and $4 \mathrm{~K}$ cooled down by a pulse tube cryocooler. Another shield, cooled at $1 \mathrm{~K}$ by a $\mathrm{He}^{4}$ fridge, surrounds the focal plane assembly. The detectors reach $270 \mathrm{mK}$ thanks to the use of two $\mathrm{He}^{3}$ refrigerators. The external shell, made on Aluminum, is $700 \mathrm{~mm}$ diameter and $1680 \mathrm{~mm}$ high, see Figure 2. It is closed with a $190 \mathrm{~mm}$ UHMW-PE window [12]

The pulse tube is a Sumitomo RP-182B2S-F100H with $1.5 \mathrm{~W}$ at $4.2 \mathrm{~K}$ and $36 \mathrm{~W}$ at $48 \mathrm{~K}$, and uses a remote valve in order to decrease the vibrations on the focal plane. The pulse tube head is mechanically disconnected to the shell thanks to a vibrations dumper. The pulse tube is water-cooled and we plan to use $100 \mathrm{~m}$ Helium lines[13].

The sub-kelvin stages are cooled down with a Chase Twin GL10 fridge: two $\mathrm{He}^{3}$ reaching $251 \mathrm{mK}$ with loading of $20 \mu \mathrm{W}$ for the focal plane, a He $\mathrm{He}^{3}$ reaching $332 \mathrm{mK}$ with $30 \mu \mathrm{W}$ for the focal plane support and a $\mathrm{He}^{4}$ reaching $840 \mathrm{mK}$ with $150 \mu \mathrm{W}$ for the focal plane shield.

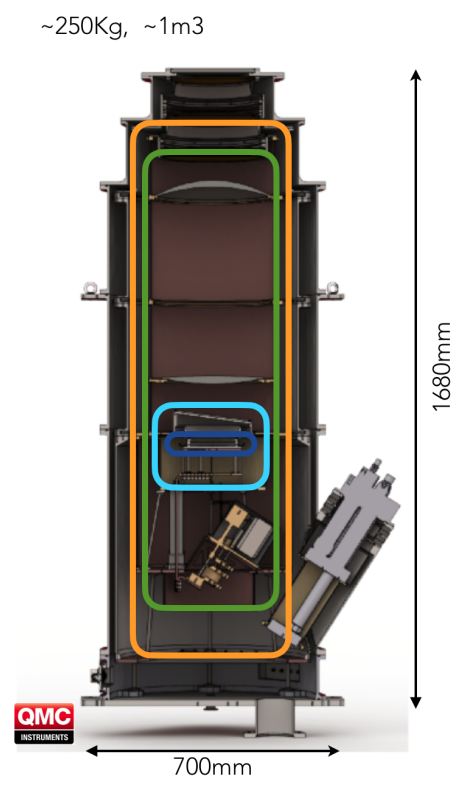

Figure 2. MISTRAL cryostat receiver provided by QMC. A pulse tube refrigerator cooled down two radiation shields, at $40 \mathrm{~K}$ (orange box) and $4 \mathrm{~K}$ (green one). The fridges cooled down the $1 \mathrm{~K}$ shield (light blue box), the focal plane support and the focal plane (dark blue box). The focal plane is cooled down at $\sim 270 \mathrm{mK}$

\subsubsection{Magnetic shield}

The experiment will move with the telescope during the observations. A magnetic shield surrounds the detectors, fridges, and relevant read-out parts to mitigate the Earth's magnetic field effects. The shield ( $1 \mathrm{~mm}$ thick) is made of Cryoperm 10 with $\mu_{r}>70000$.

\footnotetext{
${ }^{2}$ http://www.terahertz.co.uk/qmc-instruments-ltd
} 


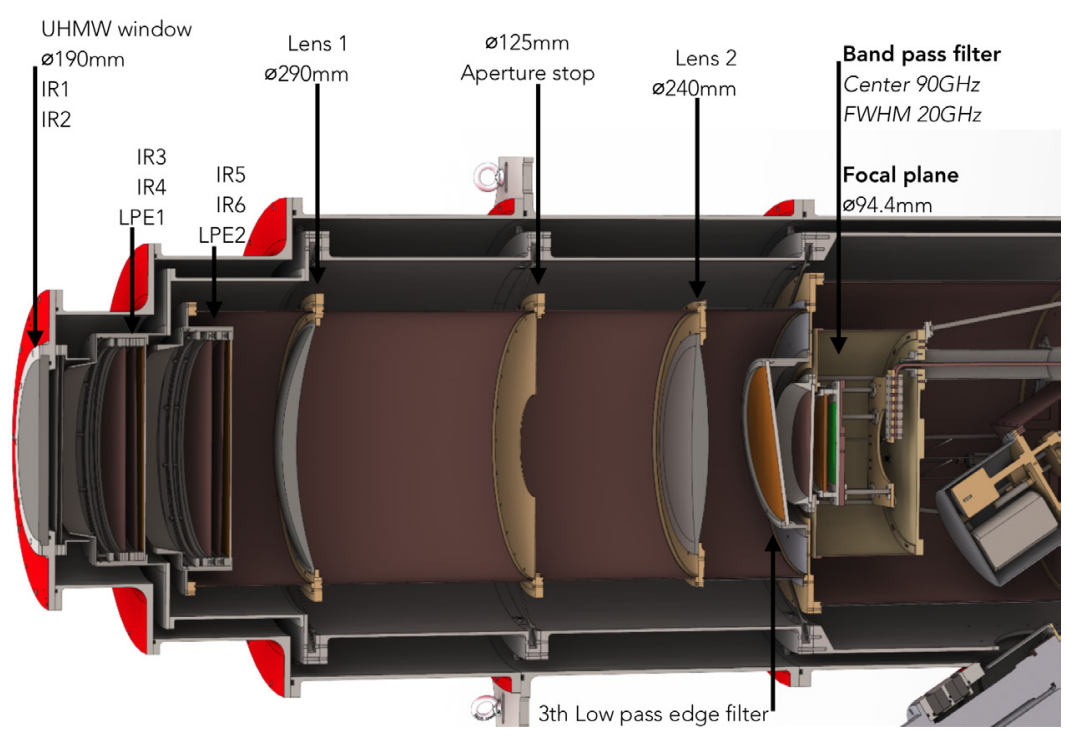

Figure 3. MISTRAL optics chain. A UHMW window closes the vacuum shell with a clearance of $190 \mathrm{~mm}$. The $40 \mathrm{~K}$ and $4 \mathrm{~K}$ shields are closed by a series of stack filters: for each shield, two thermal filters and a low pass edge filter. At $4 \mathrm{~K}$ the re-imaging optical system has been placed. The last low pass edge filter closes the $1 \mathrm{~K}$ shield. The last filter is a band-pass filter which defines the MISTRAL absorption band.

\subsection{Optics}

MISTRAL uses a re-imaging optical system, see Figure 3, to couple the Gregorian focus with the array focal plane. The re-imaging optics is composed of two Silicon lenses (L1 and L2) and a cold stop which is the aperture stop of the system. L1 is a convex-concave aspherical lens $290 \mathrm{~mm}$ in diameter. The cold stop is a $125 \mathrm{~mm}$ circular aperture coated with an absorber material (i.e. Eccosorb AN72). L2 is a convex-convex aspherical lens. The relay optics has been optimised to operate at $4 \mathrm{~K}$ assuming a Silicon refractive index of 3.4. Each lens surface will be covered with an antireflection coating based on one layer of PTFE; the efficiency in transmission is always $>90 \%$ over the MISTRAL frequency band. The field of view of the system is 4 arcmin by assuming an array focal plane $94.4 \mathrm{~mm}$ in diameter. The focal plane scale ratio is $2.54 \mathrm{arcsec} / \mathrm{mm}$. The Strehl ratio on-axis is 0.97 with an FWHM of $12.2 \mathrm{arcsec}$; at the edge of the focal plane, the Strehl ratio decrease at 0.91, and the FWHM became 12.7 arcsec. The overall efficiency of MISTRAL optics chain is $90 \%$ on-axis and decrease around $60 \%$ near the focal plane edge.

\subsection{Focal Plane}

MISTRAL focal plane will be populated with 408 LEKIDs arranged on a triangular scheme with $4.2 \mathrm{~mm}$ side. Each absorber is a front-illuminated, $3^{\text {rd }}$ order Hilbert, $3 \mathrm{~mm} \times 3 \mathrm{~mm}$ resonator, see Figure 4. The wafer is a 4 inches Silicon wafer $235 \mu \mathrm{m}$ thick, while the LEKIDs are made of superconducting Ti-Al bilayer $(10 \mathrm{~nm}+30 \mathrm{~nm})$, as in $[14,15]$. Due to the reimaging optics two close-by pixels separation is 10.6 arcsec. The band-pass filter, placed in front of the wafer, closes the holder. It is centered at $90 \mathrm{GHz}$ with $\sim 25 \mathrm{GHz}$ of bandwidth. The readout system is based on an FPGA, successfully used for OLIMPO [16]. 

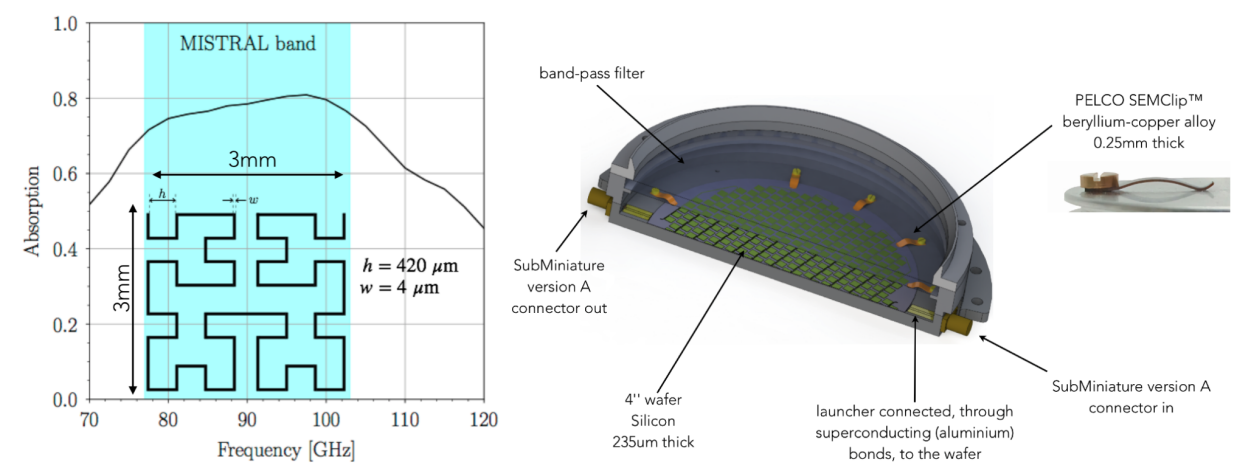

Figure 4. Left: HFSS simulations have been performed to optimize the pixel absorption. Right: The 4 inches silicon wafer is anchored inside an aluminum holder thanks to beryllium-copper spring. The holder is closed with the band-pass filter and the continuity is guaranteed with SMA connectors.

\subsubsection{Sensitivity estimation}

To predict the performance on the sky, the measured noise equivalent power (NEP) has been compared with photon noise due to warm optics and atmospheric emission, Figure 4 in [17]. The latter, should be considered in its emission part and turbulence part which will act as correlated noise. A preliminary noise equivalent power about $3.5 \times 10^{-16} \mathrm{~W} / \sqrt{\mathrm{Hz}}$ has been measured at $270 \mathrm{mK}$ by using a test-bed cryostat. By using experimental data, taken at SRT in the K-band, the atmospheric fluctuation noise, using modelling software such as $\mathrm{am}^{3}$, increases the atmospheric noise estimation a factor $\approx 10$ with respect to the photon noise level even in the best $5 \%$ percentile resulting in a noise equivalent flux density of $\approx 3 \mathrm{mJy} / \mathrm{beam}$.

\section{Conclusions}

SRT is a multipurpose observatory designed to measure a wide range of radio wavelengths: from $300 \mathrm{MHz}$ to $116 \mathrm{GHz}$. At SRT, the sky opacity in winter is $<0.15$ (50 ${ }^{\text {th }}$ percentile) at $93 \mathrm{GHz}$ MISTRAL will be coupled with SRT with a re-imaging optical system. The minimum spatial resolution (FWHM) is $12.2 \mathrm{arcsec}$ with a field of view of 4 arcmin. The 408 LEKIDs array has been optimized for the best $90 \mathrm{GHz}$ absorption and for the background at SRT.

The scientific commissioning activities will start in summer 2022.

\section{References}

[1] W.F. Wall, I. Puerari, R. Tilanus, F.P. Israel, J.E. Austermann, I. Aretxaga, G. Wilson, M. Yun, K.S. Scott, T.A. Perera et al., Monthly Notices of the Royal Astronomical Society 459, 1440 (2016), 1603.07736

[2] A. Humphrey, M. Zeballos, I. Aretxaga, D.H. Hughes, M.S. Yun, R. Cybulski, G.W. Wilson, J. Austermann, H. Ezawa, R. Kawabe et al., Monthly Notices of the Royal Astronomical Society 418, 74 (2011), 1107.3120

[3] M.S. Petersen, R.A. Gutermuth, E. Nagel, G.W. Wilson, J. Lane, Monthly Notices of the Royal Astronomical Society 488, 1462 (2019)

\footnotetext{
${ }^{3}$ https://www.cfa.harvard.edu/ spaine/am/
} 
[4] A. Sokol, R. Gutermuth, R. Pokhrel, A. Gómez-Ruiz, G. Wilson, S. Offner, M. Heyer, A. Luna, F. Schloerb, D. Sánchez, Monthly Notices of the Royal Astronomical Society 483, 407 (2019)

[5] M.J. Hardcastle, L.W. Looney, Monthly Notices of the Royal Astronomical Society 388, 176 (2008), 0804. 3369

[6] T. Mroczkowski, D. Nagai, K. Basu, J. Chluba, J. Sayers, R. Adam, E. Churazov, A. Crites, L. Di Mascolo, D. Eckert et al., Space Science Reviews 215, 17 (2019), 1811.02310

[7] A. de Graaff, Y.C. Cai, C. Heymans, J.A. Peacock, Astronomy \& Astrophysics 624, A48 (2019), 1709.10378

[8] S.R. Dicker, P.A.R. Ade, J. Aguirre, J.A. Brevik, H.M. Cho, R. Datta, M.J. Devlin, B. Dober, D. Egan, J. Ford et al., Journal of Low Temperature Physics 176, 808 (2014)

[9] P. Bolli, A. Orlati, L. Stringhetti, A. Orfei, S. Righini, R. Ambrosini, M. Bartolini, C. Bortolotti, F. Buffa, M. Buttu et al., Journal of Astronomical Instrumentation 04, 1550008 (2015), https: //doi .org/10.1142/S2251171715500087

[10] F. Govoni et al., 2021 XXXIVth General Assembly and Scientific Symposium of the International Union of Radio Science -, 1 (2021)

[11] F.T. Nasir, F. Buffa, G.L. Deiana, Experimental Astronomy 29, 207 (2011)

[12] G. D’Alessandro, A. Paiella, A. Coppolecchia, M. Castellano, I. Colantoni, P. de Bernardis, L. Lamagna, S. Masi, Infrared Physics \& Technology 90, 59 (2018)

[13] A. Coppolecchia, E. Battistelli, Masi et al., Pulse tube cooler with $>100 \mathrm{~m}$ flexible lines optimized for operation of cryogenic detector arrays at large radiotelescopes (2021)

[14] Catalano, A., Goupy, J., le Sueur, H., Benoit, A., Bourrion, O., Calvo, M., D’addabbo, A., Dumoulin, L., Levy-Bertrand, F., Macías-Pérez, J. et al., Astronomy \& Astrophysics 580, A15 (2015)

[15] A. Paiella, A. Coppolecchia, M.G. Castellano, I. Colantoni, A. Cruciani, A. D'Addabbo, P. de Bernardis, S. Masi, G. Presta, Journal of Low Temperature Physics 184, 97 (2016)

[16] A. Paiella, E.S. Battistelli, M.G. Castellano, I. Colantoni, F. Columbro, A. Coppolecchia, G. D’Alessandro, P. de Bernardis, S. Gordon, L. Lamagna et al., Journal of Physics: Conference Series 1182, 012005 (2019)

[17] A. Coppolecchia, A. Paiella, L. Lamagna, G. Presta, E.S. Battistelli, P. de Bernardis, M.G. Castellano, F. Columbro, S. Masi, L. Mele et al., Journal of Low Temperature Physics 199, 130 (2020) 